\title{
Front Dynamics with Time Delays in a Bistable System of the Reaction-Diffusion Type: Role of the Symmetry of the Rate Function
}

\author{
R. BAKAnAS ${ }^{a, *}$, F. IVANAUSKAS ${ }^{b, c}$ AND V. JASAitis ${ }^{b}$ \\ ${ }^{a}$ Semiconductor Physics Institute, A. Goštauto 11, 2600 Vilnius, Lithuania \\ ${ }^{b}$ Faculty of Mathematics and Informatics, Vilnius University, Naugarduko 24, LT-03225 Vilnius, Lithuania \\ ${ }^{c}$ Institute of Mathematics and Informatics, Akademijos 4, 2600 Vilnius, Lithuania \\ (Received January 28, 2010; revised version April 28, 2010; in final form October 24, 2010)
}

\begin{abstract}
The retardation effects in dynamics of the ac driven "bistable" fronts joining two states of the different stability in a bistable system of the reaction-diffusion type are investigated by use of the macroscopic kinetic equation of the reaction kinetics. We approximate the rate (reaction) function in the governing equation of "bistable" fronts by the piecewise linear dependence of the flexible symmetry, encompassing both cases of the symmetrical and asymmetrical rate functions. By numerically simulating the drift motion of the ac driven front being subjected to the time-dependent step-like (rectangular) forcing we investigate the lag time between the ac force and the instantaneous velocity of the ac driven front. We find that the time lags derivable by the symmetrical and asymmetrical rate functions notably differ, namely, we show that (a) the lag time is a function of the outer slope coefficients of the rate function and is not sensitive to the inner, (b) it has only weak dependence on the strength of the applied forcing, (c) the retardation effects (time lags) in the front dynamics are describable adequately enough by use of the perturbation theory. Another aspect of the front dynamics discussed in this report is the influence of the retardation effects on the ratchet-like transport of the ac driven fronts being described by the asymmetrical rate functions of the "low" symmetry. By considering the response of "bistable" front to the single-harmonic ac force we find that the occurrence of the time lags in the oscillatory motion of the ac driven front shrink the spurious drift of the front; the spurious drift practically disappears if the frequency of the oscillatory force significantly exceeds the characteristic relaxation rate of the system. Furthermore, the occurrence of the time lags in the front dynamics leads to the vanishing of the reversals in the directed net motion of the ac driven fronts, being always inherent in the case of the slow (quasi-stationary) ac drive, i.e., the possibilities of controlling the directed net motion of the self-ordered fronts by the low- and high-frequency zero-mean ac forces radically differ.
\end{abstract}

PACS: 05.45.-a, 02.30.Jr, 82.40.Ck

\section{Introduction}

The "unforced" direct transport of both the point-particles (electrons, atoms, molecules) and the selfordered front-structures without any net external force (the so-called ratchet effect) has today become a fundamental field of multidisciplinary research in a wide variety of systems driven out of thermal equilibrium. A number of theoretical and experimental studies in recent years have been devoted to the ratchet effects both, for point particles and the self-ordered fronts, both the coherent and dissipative ones (see Refs. [1] and [2-12], respectively). The ratchet-like transport implies that a steady, systematic direct motion of the particle/self-ordered front being subjected to the oscillatory force of zero-time average, either noisy or regular one, takes place. The crucial feature of the systems performing the unforced dc transport is the presence of dissipation, which implies that the ac driven system escapes its memory when the development of the system progresses. The ratchet-like transport of the dissipative fronts that result

* corresponding author; e-mail: bakanas@pfi.lt from the competition between the nonlinearity and diffusion in the essentially dissipative systems of the reaction-diffusion type has been studied in numerous papers, both numerically and by analytic tools (see Refs. [3-12]). Two different kinds of the self-ordered fronts, namely, the "bistable" fronts (BFs) joining the stable and metastable states of the system, and "monostable" ones that separate the stable and unstable states of the system have been analyzed (e.g., see reviews [13], for the self-ordered fronts in reaction-diffusion systems). An intriguing property of the systems performing the ratchet-like shuttling of the self-ordered fronts is their capability to rectify the oscillatory motion of the ac driven front even in the case of the spatially uniform system, by contrast to the ordinary ratchets performing the unforced dc motion of the free particles in the systems lacking the spatial inversion symmetry. The unforced dc drift of the self-ordered fronts usually originates due to the "hidden" asymmetry of the system, or it comes from the broken temporal symmetry of the oscillatory force acting on the front in the system. Two different mechanisms underlying the spurious drift discussed have been identified, namely, the parametrically stimulated and directly induced dc drift of the fronts (e.g., see Refs. [3-5] and [6-10], respectively). The 
parametrically stimulated dc drift comes through the action of the external oscillatory field of zero-time average on the externally controllable parameter of the system: the external time-symmetric field acting on the system is transformed into the asymmetrically oscillating force of a finite time average, that pushes the front in the system (e.g., see Refs. $[3-5]$ ). By contrast, the directly induced dc drift implies that the average force $f(t)$ acting on the front in the system equals zero. The evolution equation of the directly driven front reads

$$
u_{t}-u_{z z}-c(t) u_{z}+R(u)=f(t),
$$

where the function $u(z, t)$ denotes the step-like field of the front propagating at the instantaneous velocity $c(t)$, and the notation $z=x-\chi(t)$ stands for the traveling (co-moving) coordinate, where $\chi(t)=\int_{t_{0}}^{t} d t^{\prime} c\left(t^{\prime}\right)$, with $t_{0}$ denoting the time moment, at which both the traveling frame of reference and that being at rest coincide. The rate (reaction) function $R(u)$ that characterizes the rate of the transient processes in the system has three zeroes at $u=u_{1}, u_{2}, u_{3}$ (say, $u_{1}<u_{2}<u_{3}$ ), more specifically, the following relations hold: $R^{\prime}\left(u_{1,3}\right)>0$ and $R^{\prime}\left(u_{2}\right)<0$ where the prime denotes the derivative. The outer zeropoints of the rate function, $u_{1}$ and $u_{3}$, describe two steady states of the system, the stable and metastable one, and the inner $u_{2}$ stands for unstable one.

In the present report we shall deal with the "bistable" fronts. The free, unperturbed $(f \equiv 0)$ front-solution $u_{0}(z)$ of $\mathrm{BF}$ joins the outer zero-points of the rate function, $u_{1}$ and $u_{3}$. Without loss of generality, we shall take in what follows that $u_{0}(z \rightarrow-\infty) \rightarrow u_{1}$, and $u_{0}(z \rightarrow \infty) \rightarrow u_{3}$. As mentioned, the "front-ratchet" is capable to operate even under spatially uniform conditions; the governing equation of the ac driven front (1) satisfies the translational symmetry with respect to the infinitesimal translations of the spatial coordinate $z$; the structural (intrinsic) asymmetry of the system is masked in the rate function. The symmetry properties of $R-u$ dependence play a prominent role, in considering the ratchet-like transport of BFs, as shown in Refs. [8, 10]. For instance, both the oscillatory speed functions $c(t)$ and the average characteristics of the spurious drift of the ac driven $\mathrm{BF}$ derivable by the symmetrical and asymmetrical rate functions satisfying the different $R-u$ symmetry (see below), radically differ. The progressive (accelerated), regressive (decelerated) and reversal types of the unforced dc motion of BFs under slow (quasi-stationary) ac force have been identified by use of the asymmetrical rate functions of the "low" $R-u$ symmetry, by contrast to the other case of the symmetrical rate functions (see Refs. $[7,8])$. The governing equation of the quasi-stationary driven $\mathrm{BF}$ reads

$$
\begin{aligned}
& u_{z z}+c(t) u_{z}-R_{\mathrm{F}}(u)=0, \\
& R_{\mathrm{F}}(u)=R(u)-f(t) .
\end{aligned}
$$

The presented evolution Eq. (2), below referred to as the adiabatic approximation of the governing Eq. (1), is very approximate. It ignores the time lags (retardation effects) between the ac force $f(t)$ and the speed function $c(t)$ of the ac driven BF, i.e., it describes the immediate, instantaneous response of $\mathrm{BF}$ to the applied force. Clearly, the instantaneous velocity ac driven BF follows behind the time-dependent forcing with some lag (retardation); the actual speed functions $c(t)$ are retarded with respect to the applied forcing $f(t)$. Our previous calculations being performed within the pseudolinear (piecewise linear) model of the system, by use of the symmetrical rate functions demonstrated that the retardation effects in the front dynamics shrunk the spurious drift discussed; the spurious drift of BF practically disappeared if the frequency of the ac force acting on the front in the system exceeded the characteristic relaxation rate $\tau_{\mathrm{R}}^{-1}$ of the system (see Refs. [11, 12]). By contrast, the quasistationary ac drive is of the highest "efficiency" in terms of the speed rectification discussed; it always induces the maximal shift of the mean drift velocity $\langle c(t)\rangle$ of the ac driven front, as shown in Ref. [12]. Both the symmetry of the rate function and the retardation effects discussed play very important role, in considering the ratchet-like transport of BFs.

In the present report the retardation effects in dynamics of the ac driven BFs being described by the asymmetrical rate functions are investigated. The pseudolinear model of the system is used, namely, we approximate the rate function by the piecewise linear $R-u$ dependence of the flexible symmetry. The piecewise linear rate function provides us with a possibility of controlling the characteristic relaxation rates of the system, in considering the retarded accelerations of the ac driven BFs. The piecewise linear emulations of the rate function have been used in the previous studies of the self-ordered fronts in multistable systems of the different physical origin (e.g., see Refs. [14] and references therein). The present report is further development and generalization of our previous results being derived in the particular case of the symmetrical rate functions (see Refs. [11, 12]). We investigate the dynamical properties of the ac driven BFs numerically and, partially, also analytically. Namely, the particular case of the slow ac drive that describes immediate, instantaneous response of BF to the applied forcing is studied analytically, by use of Eq. (2), and the other case of the fast driving, the front dynamics with a delayed, postponed reaction of BF to the ac drive was studied numerically, by the direct solution of the governing Eq. (1).

The paper has two distinct aims. The first, which we address in Sect. 3.2, is to investigate the retardation effects in the front dynamics. Namely, the lag time between the ac force $f(t)$ and the speed function $c(t)$ of the ac driven BFs derivable by the piecewise linear rate function of the flexible $R-u$ symmetry is studied. In considering the time lags we approximate the forcing function by the step-like (rectangular) $f-t$ dependence. By considering the response of $\mathrm{BF}$ to the rectangular forcing we show that the temporal behavior of the retarded speed 
functions $c(t)$ is exponential. Furthermore, we find that the characteristic time duration $\tau_{S}$ denoting the lag time between the step-like speed function $c(t)$ and the rectangular forcing is describable adequately enough by use of the perturbation theory, even in the case of the strong forcing.

Another aspect of the front dynamics, discussed in the present report is the influence of the retardation effects on the spurious drift of BFs derivable by the asymmetrical rate functions. In particular, we investigate whether and how strongly the time lags discussed are capable to influence on the reversal behavior of the directed net motion of the ac driven fronts derivable by the asymmetrical rate functions. In considering the ratchet-like shuttling of BFs we approximate the oscillatory force $f(t)$ acting on the front in the system by the single-harmonic forcing function. The average characteristics of the spurious drift of the "retarded" BFs are presented. The retardation effects in dynamics of the ac driven BFs that are described by the asymmetrical rate functions of the low $R-u$ symmetry, as far as we know, have not previously been studied elsewhere in the literature.

The paper is organized as follows. In Sect. 2 we discuss the model, approximations and techniques used. Section 3 deals with the retarded speed functions of the ac driven BFs being under the action of the time-dependent rectangular forcing. In Sect. 4 the ratchet-like transport of the periodically forced BFs is investigated. Finally, in Sect. 5 we summarize the main results.

\section{Model, approximations and techniques used}

Before discussing the model and the techniques used, let us touch briefly on the relevant symmetries of the rate functions used (for more details, see Ref. [10]). We shall deal with the self-similar rate functions being defined by the relation:

$$
R(u ; C):=R_{0}(u)+C,
$$

where $C$ is the free constant and the subscript zero refers to the balanced rate function $R_{0}(u)$ satisfying the relation (Maxwellian construction) $S_{0} \equiv \int_{u_{1}}^{u_{3}} \mathrm{~d} u R_{0}(u)=0$, where the notation $S_{0}$ stands for the total area subtracted by $R_{0}-u$ dependence within the interval $\left[u_{1}, u_{3}\right]$ of the variable $u$. Without loss of generality, two different classes (families) of the symmetrical (symmetrically shaped) and asymmetrical (asymmetrically shaped) rate functions $R(u ; C)$ satisfying the relations

$$
R_{0}\left(u-u_{2}\right)=-R_{0}\left(u+u_{2}\right)
$$

and

$$
R_{0}\left(u-u_{2}\right) \neq-R_{0}\left(u+u_{2}\right),
$$

respectively, may be typified. Both families discussed encompass the most general case of the system with two stable equilibria. In the present report we shall deal with the asymmetrical rate functions $R_{\mathrm{A}}(u)$. The asymmetrical rate function, $R_{\mathrm{A}}(u ; C):=R_{0}(u)+C$, implies that the basic (balanced) rate function is asymmetrically shaped with respect to the inner zero-point $u_{2}$, i.e., the inequality (4b) holds. For brevity, in what follows we shall omit the subscript "A" in the notation $R_{\mathrm{A}}(u)$.

Obviously, the free constant $C$ cannot exceed the critical value: the criterion of the global stability of $\mathrm{BF}$ reads, $C \in\left(-R_{0 \mathrm{M}},-R_{0 \mathrm{~m}}\right)$ where by $R_{0 \mathrm{M}}$ and $R_{0 \mathrm{~m}}$ we denote the extremes of the balanced rate function, and the subscripts $\mathrm{M}$ and $\mathrm{m}$ denote "Maximal" and "minimal", respectively. By tuning the free constant $C$ in Eq. (3) one governs Maxwellian construction of the rate function $R(u ; C)$, as a consequence, one arrives at the different propagation rates $c_{0}$ of the free, unperturbed $\mathrm{BF}$. The free $\mathrm{BF}$ is static (motionless, $c_{0}=0$ ) if the Maxwellian construction is balanced, namely, if one takes that $C=0$ (e.g. see Refs. [7-9, 16]). Let us turn to the model and the techniques used.

\subsection{Model}

We shall deal with the piecewise linear rate functions being constructed from three linear pieces, thus we write that (see Fig. 1)

$$
R(u)= \begin{cases}\alpha_{1}\left(u-u_{1}\right), & u<u_{\mathrm{M}}, \\ -\alpha_{2}\left(u-u_{2}\right), & u_{\mathrm{M}}<u<u_{\mathrm{m}}, \\ \alpha_{3}\left(u-u_{3}\right), & u>u_{\mathrm{m}},\end{cases}
$$

where the free parameters $u_{i}$ and $\alpha_{i}$ satisfy the relations $u_{1}<u_{\mathrm{M}}<u_{2}<u_{\mathrm{m}}<u_{3}$ and $\alpha_{i}>0(i=1,2,3)$, and the extremes of the rate function, $R_{\mathrm{M}} \equiv R\left(u_{\mathrm{M}}\right)$ and $R_{\mathrm{m}} \equiv R\left(u_{\mathrm{m}}\right)$, are described as follows, $R_{\mathrm{M}, \mathrm{m}}=$ $\alpha_{2}\left(u_{2}-u_{\mathrm{M}, \mathrm{m}}\right)$. The piecewise linear rate function used (a) is analytically tractable; both the free front solutions of BFs and the perturbed ones derivable by use of Eq. (2), within the adiabatic approximation discussed, are known explicitly (see Ref. [8, 15]), (b) is of the flexible $R-u$ symmetry; by taking $\alpha_{1}=\alpha_{3}$ and $\alpha_{1} \neq \alpha_{3}$ one arrives at both cases of the symmetrical and asymmetrical rate functions, respectively, (c) reproduces qualitatively well enough the characteristic features of the bistable system; both the speed functions $c(t)$ and the average characteristics of the spurious drift of BFs derivable by use of both the prototype (cubic polynomial) rate function and its piecewise linear emulations (5) satisfying the same $R-u$ symmetry are very close to each other, as shown in Refs. $[10,12]$.

In the present report the general case of the asymmetrical rate functions is considered; we do not impose any additional restrictions on the rate function (5). For our purposes it is useful to introduce the auxiliary parameters, $h_{0}=-R_{0 \mathrm{M}} / R_{0 \mathrm{~m}}$ and $h_{\mathrm{R}}=-R_{\mathrm{M}} / R_{\mathrm{m}}$, below referred to as "balance parameters" of the balanced and unbalanced rate functions, $R_{0}(u)$ and $R(u)$, respectively. The Maxwellian construction of the rate function (5) is balanced (at the Maxwell point) if one takes that

$$
h_{0}=\sqrt{\left(1+\delta_{3}\right) /\left(1+\delta_{1}\right)}, \quad \delta_{1,3}=\alpha_{2} / \alpha_{1,3} .
$$

The unbalanced rate function $R(u ; C)$ implies that 


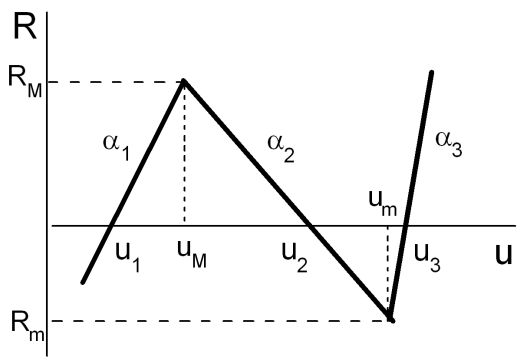

Fig. 1. The piecewise linear rate function.

$h_{\mathrm{R}} \neq h_{0}$ (or equivalently, $\left.C \neq 0\right)$; the obvious relation $h_{\mathrm{R}}=-\left(R_{0 \mathrm{M}}+C\right) /\left(R_{0 \mathrm{~m}}+C\right)$ holds. By tuning the balance parameter $h_{\mathrm{R}}$ one governs the propagation velocity of the free BF. The maximal propagation rate of the forward $\left(c_{0}>0\right)$ and the backward $\left(c_{0}<0\right)$ running BFs is achieved by taking the limits $h_{\mathrm{R}} \rightarrow \infty$ and $h_{\mathrm{R}} \rightarrow 0$, respectively. More specifically, the following relations hold: (a) $c_{0} \rightarrow c_{\mathrm{M}}$ if $h_{\mathrm{R}} \rightarrow \infty$, and (b) $c_{0} \rightarrow-c_{\mathrm{M}}$ if $h_{\mathrm{R}} \rightarrow 0$ where the notation $c_{\mathrm{M}}$ stands for the maximal (marginal) velocity of $\mathrm{BF}$, namely, one has that $c_{\mathrm{M}}=2 \sqrt{\alpha_{2}}$ (the boundary conditions $u_{0}(z \rightarrow \mp \infty) \rightarrow u_{1,3}$ discussed above are taken into account in the derivation of these inequalities (e.g., see Ref. [8])). In what follows we shall use the scaled speed functions $s(t)$ being defined by the relation $s(t):=c(t) / c_{\mathrm{M}}$. The scaled speed functions are preferable over the ordinary (non-scaled) ones. They take the values within the interval $(-1,1)$, namely, the relation $s(t) \in(-1,1)$ holds in any case, regardless to the balance parameter value $h_{\mathrm{R}}$ used (see Refs. [10, 12]). Quite similarly, the scaled velocity of the free, unperturbed $\mathrm{BF}, s_{0}:=c_{0} / c_{\mathrm{M}}$, takes values within the interval $(-1,1)$, more specifically, the following relations hold: $s_{0} \rightarrow 1$ if $h_{\mathrm{R}} \rightarrow \infty$ and $s_{0} \rightarrow-1$ if $h_{\mathrm{R}} \rightarrow 0$.

Both the heights, $\Delta R:=R_{\mathrm{M}}\left(u_{\mathrm{M}} ; C\right)-R_{\mathrm{m}}\left(u_{\mathrm{m}} ; C\right)$, of the self-similar rate functions $R(u ; C)$ and the positions of the extremes, being located at $u=u_{\mathrm{M}}$ and $u=u_{\mathrm{m}}$ do not depend on the free constant $C$. From Eq. (5), if used in conjunction with (3), follows that

$$
\begin{aligned}
& R_{\mathrm{M}}=h_{\mathrm{R}}\left(1+h_{\mathrm{R}}\right)^{-1} \Delta R, \\
& R_{\mathrm{m}}=-\left(1+h_{\mathrm{R}}\right)^{-1} \Delta R, \\
& u_{1}=u_{\mathrm{M}}-\alpha_{1}^{-1} R_{\mathrm{M}}, \quad u_{2}=u_{\mathrm{M}}-\alpha_{2}^{-1} R_{\mathrm{M}}, \\
& u_{3}=u_{\mathrm{m}}-\alpha_{3}^{-1} R_{\mathrm{m}} .
\end{aligned}
$$

In closing the discussion of the piecewise linear rate functions we notice that the free front-solutions of BFs, $u_{0}(z)$, derivable by the rate function of the flexible symmetry (5) are presented in Ref. [15]. Let us touch briefly on the techniques used.

\subsection{Techniques used}

The governing equation of the ac driven $\mathrm{BF}(1)$ is not solvable, even in the case of the piecewise linear rate function (5). In view of this, in considering the dynamical properties of the ac driven BFs we used both the numerical and analytic tools. As previously mentioned, the particular case of the slowly driven BFs, that describes the immediate, instantaneous response of $\mathrm{BF}$ to the applied forcing was studied analytically, by use of Eq. (2), and the other case of the rapid driving, the front dynamics with a delayed response of $\mathrm{BF}$ to the ac force was studied numerically, by use of Eq. (1). The governing equation of the quasi-stationary driven BF (2) bears a close similarity to that of the free, unperturbed BF; both the speed functions and the front solutions of the slowly driven BFs derivable by the piecewise linear rate function (5) are known explicitly (see Ref. [8]). The scaled speed functions $s(t)$ are the main subject of the present study. The "instantaneous" speed functions $s_{\mathrm{A}}(t)$ that ignore the time lags discussed are derivable by use of the "speed equation" being derived by the direct solution of Eq. (2). The speed equation reads (see Ref. [8] for details)

$$
\frac{\Psi_{S n}\left(s_{\mathrm{A}}\right)}{\exp \left(-\varphi\left(s_{\mathrm{A}}\right)\right) \sin \Phi\left(s_{\mathrm{A}}\right)}=\frac{h_{\mathrm{R}}-\left(1+h_{\mathrm{R}}\right) f(t)}{1+\left(1+h_{\mathrm{R}}\right) f(t)},
$$

where the auxiliary functions are defined by the relations

$$
\begin{aligned}
& \varphi\left(s_{\mathrm{A}}\right)=\frac{s_{\mathrm{A}} \Phi\left(s_{\mathrm{A}}\right)}{Q_{2}\left(s_{\mathrm{A}}\right)}, \\
& \Phi\left(s_{\mathrm{A}}\right)= \begin{cases}\arctan \Psi_{T g}\left(s_{\mathrm{A}}\right), & \Psi_{T g}\left(s_{\mathrm{A}}\right)>0, \\
\pi-\arctan \left[-\Psi_{T g}\left(s_{\mathrm{A}}\right)\right], & \Psi_{T g}\left(s_{\mathrm{A}}\right)<0,\end{cases}
\end{aligned}
$$

and the unknown functions are described as follows:

$$
\begin{aligned}
& \Psi_{S n}\left(s_{\mathrm{A}}\right)=F_{S n} / F_{V}, \quad \Psi_{T g}\left(s_{\mathrm{A}}\right)=F_{S n} / F_{C n}, \\
& F_{S n}=Q_{2}\left(s_{\mathrm{A}}\right)\left[\delta_{1} K_{1}\left(s_{\mathrm{A}}\right)-\delta_{3} K_{3}\left(s_{\mathrm{A}}\right)\right], \\
& F_{C n}=-\left[Q_{2}^{2}\left(s_{\mathrm{A}}\right)+G_{1}\left(s_{\mathrm{A}}\right) G_{3}\left(s_{\mathrm{A}}\right)\right], \\
& F_{V}=Q_{2}^{2}\left(s_{\mathrm{A}}\right)+G_{1}^{2}\left(s_{\mathrm{A}}\right), \\
& G_{1,3}=-s_{\mathrm{A}}+\delta_{1,3} K_{1,3}\left(s_{\mathrm{A}}\right),
\end{aligned}
$$

where

$$
\begin{aligned}
& Q_{2}\left(s_{\mathrm{A}}\right)=\sqrt{1-s_{\mathrm{A}}^{2}}, \\
& K_{1,3}\left(s_{\mathrm{A}}\right)=-s_{\mathrm{A}} \pm \sqrt{r_{1,3}+s_{\mathrm{A}}^{2}},
\end{aligned}
$$

and by $\delta_{1,3}$ and $r_{1,3}$ we denote the characteristic parameters described by the relation $r_{1,3}=\delta_{1,3}^{-1}:=\alpha_{1,3} / \alpha_{2}$. The boundary conditions $u_{0}(z \rightarrow \mp \infty) \rightarrow u_{1,3}$ discussed above have been used in the derivation of these equations. To avoid the confusion we stress that the denotation $s_{\mathrm{A}}(t)$ stands for the "instantaneous" speed function, throughout this paper.

In considering the retarded accelerations of BFs, the front dynamics with the time lags, we numerically simulated the drift motion of the ac driven $\mathrm{BF}$. To find both the front-solution $u(z, t)$ and the retarded speed function $s(t)$ of the ac driven BF we numerically solved the gov- 
erning Eq. (1) in the co-moving uniform grid. Equation (1) was approximated by the finite difference scheme in the co-moving coordinates, $z=x-x_{C}(t)$, with the traveling center of the ac driven front, $x_{C}(t)$, being defined by the relation $x_{C}(t)=\Delta u^{-1}(t) \int_{-\infty}^{\infty} \mathrm{d} x x u_{x}(x, t)$ where, $\Delta u(t)=\int_{-\infty}^{\infty} \mathrm{d} x u_{x}(x, t)$. The speed functions $s(t)$ of the ac driven BFs have been obtained by considering the traveling center $x_{C}(t)$ of the front.

Finally, let us specify the forcing functions used. As discussed, in considering the time lags between the ac force and the instantaneous velocity $s(t)$ of the ac driven $\mathrm{BF}$ we approximated the forcing function by the step-like $f-t$ dependence,

$$
F(t)=F_{0} \Theta\left(t-t_{0}\right),
$$

where the denotation $\Theta(x)$ stands for the Heaviside unit-step function, and by $F_{0}$ and $t_{0}$ we denote the strength of the rectangular forcing and the initial moment, at which the step-like force was switched on, respectively. The forcing function used is extremely fast (steep); it provides us with a possibility of testing a wide "spectrum" of the characteristic relaxation rates of the temporal (transient) behavior of the retarded speed functions $s(t)$.

As mentioned, another aspect of the front dynamics discussed in this report is the influence of the time lags discussed on the ratchet-like transport of BFs drivable by the asymmetrical rate functions. To reveal the role of $R-u$ symmetry in the dynamics of the rectified oscillatory motion of BFs, we used the time-symmetric forcing function. Namely, the response of BF to the single-harmonic forcing function,

$$
f(t)=f_{0} \sin \omega t,
$$

was investigated. The forcing function (10) is of the "highest" temporal symmetry. It satisfies both the time-shift and time-inversion symmetry relations, $f(t+$ $T / 2)=f(t)$ and $f\left(t_{N}-t\right)=-f\left(t_{N}+t\right)$, respectively, where the notation $t_{N}$ stands for the $N$-th zero-point of the oscillatory function $f(t)$, namely, one has that $t_{N}=$ $2 N \pi$ where $N=0, \pm 1, \pm 2, \ldots$ Obviously, the strength of the driving force acting on the front in the system cannot exceed the critical value, $f_{\mathrm{M} x}=\min \left\{R_{\mathrm{M}},-R_{\mathrm{m}}\right\}$; the criterion of the global stability of the ac driven BF reads, $\left(f_{0}, F_{0}\right)<f_{\mathrm{M} x}$.

\section{Front dynamics with time delays}

\subsection{Preliminary remarks: perturbative approach}

The lag time $\tau_{D}$ between the ac force $f(t)$ and the speed function $s(t)$ of the ac driven BF is sensitive to both rates that characterize the driving force and the system. More specifically, it depends on both the steepness of the forcing function, that characterizes the "rate" of the driving force, and the characteristic relaxation time of the system, $\tau_{\mathrm{R}}$, which describes the rate of the transient processes in the system (e.g., see Ref. [11]). Regretfully, the characteristic parameter $\tau_{\mathrm{R}}$ is analytically not obtainable; the analytical techniques of finding the front-solutions of the ac driven $\mathrm{BF}$ are lacking. The previous investigations of the ac driven BFs have been based, for the most part, on the adiabatic approximation (2) ignoring the time lags discussed. Nevertheless, a rough estimate of the required parameter $\tau_{\mathrm{R}}$ may be obtained by use of the perturbation theory that describes the particular case of the weakly perturbed system being only slightly deviated from its stationary "state" $u_{0}(z)$. As a preliminary, it seems to be instructive to begin our consideration of the retardation effects in the front dynamics with a brief discussion of the characteristic relaxation rates $\tau_{\mathrm{R}}^{-1}$ in a weakly perturbed system. The temporal behavior of the system being only slightly deviated from its stationary state $u_{0}(z)$ is obtainable through the linearization procedure of the governing Eq. (1), in which we replace the forcing function $f(t)$ by the weak forcing $\varepsilon f(t, z)$, where $\varepsilon \ll 1$. Then, it follows that $u(z, t)=u_{0}(z)+\Delta u(z, t)$ and $c(t)=c_{0}+\Delta c(t)$ where by $\Delta u(z, t)$ and $\Delta c(t)$ we denote the small deviations. With the linearization procedure discussed the following equation results (e.g., see Refs. [5, 17]):

$$
\begin{gathered}
\Delta u_{t}(z ; t)+\hat{L} \Delta u(z ; t)-\Delta c(t) u_{0 z} \\
=f(t, z)+O\left[\Delta u, \Delta c ; u_{0}(z)\right],
\end{gathered}
$$

where the linear operator $\hat{L}$ is defined as follows:

$$
\hat{L}=-\partial_{z z}-c_{0} \partial_{z}+U(z),
$$

and the associated potential $U(z)$ is described by the relation

$$
U(z)=R^{\prime}\left[u_{0}(z)\right],
$$

with the prime denoting the derivative with respect to the "variable" $u_{0}$. The auxiliary function $O(z, t)$ in Eq. (11) involves the nonlinear terms in $\Delta u$ and $\Delta c$. Equation (11) holds in any case the weak forcing $\varepsilon f(t, z)$, either noisy or regular one, independently of the initial cause that produced the small deviations $\Delta u$ and $\Delta c$. From Eq. (11) it follows that the temporal behavior of the system being only slightly deviated from its stationary state $u_{0}(z)$ may be evaluated by solving the following eigenvalue problem (e.g., see Refs. [5, 17] for details):

$$
\hat{L} Y_{\alpha}(z)=\lambda_{\alpha} Y_{\alpha}(z),
$$

where the notations $Y_{\alpha}$ and $\lambda_{\alpha}$ stand for the eigenfunction and the corresponding eigenvalue of the linear operator $\hat{L}$, respectively. The eigenvalues spectrum $\left\{\lambda_{\alpha}\right\}$, which encompasses an ordered set of the eigenvalues $\lambda_{\alpha}$ denoting the characteristic relaxation rates (exponents), $\tau_{\alpha}^{-1}=\lambda_{\alpha}$, of a weakly perturbed system, depends on the peculiarities of the potential $U(z)$ being a function of both the rate function $R(u)$ and the front solution $u_{0}(z)$, as follows from Eqs. (13), (12a) and (12b). The free front solution of $\mathrm{BF}, u_{0}(z)$, joins the outer zero points of the rate function. Thus, from Eq. (12b) it follows that the potential function $U(z)$ describes the potential well with two maxima being at infinity, $z \rightarrow \mp \infty$, and 
the minimum being located at the point $z=z_{\mathrm{m}}$ derivable from the following equation: $u_{0}\left(z_{\mathrm{m}}\right)=u_{\mathrm{f}}$ where the denotation $u_{\mathrm{f}}$ stands for the flex-point of the rate function, namely, one has that $R^{\prime \prime}\left(u_{\mathrm{f}}\right)=0$. The obvious relations that are followed directly from Eq. (12b) hold: $U(z \rightarrow \mp \infty) \rightarrow U_{\mp \infty}$ where $U_{\mp \infty}=R^{\prime}\left(u_{1,3}\right)$. Referring to the particular case of the pseudolinear rate function studied in the present report we get that $U_{-\infty}=\alpha_{1}$, $U_{+\infty}=\alpha_{3}$, and $U\left(z_{\mathrm{m}}\right)=-\alpha_{2}$, i.e., the potential function $U(z)$ describes the square well being schematically depicted in Fig. 2.

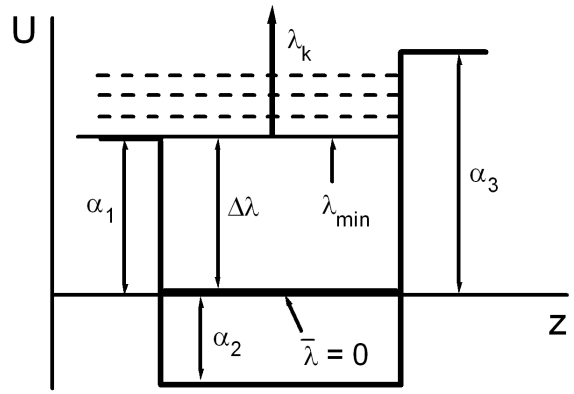

Fig. 2. The associated potential of the linearized problem being related to the pseudolinear model of the bistable system.

In continuing the discussion of the characteristic relaxation rates $\tau_{\alpha}^{-1}$ we notice that the eigenvalues spectrum $\left\{\lambda_{\alpha}\right\}$ has a gap. More specifically, it contains at least one discrete (separate) eigenlevel, $\bar{\lambda}=0$, being related to the translational (Glodstone) mode, $\bar{Y}(z) \propto u_{0}^{\prime}(z)$, and the continuous spectrum of the eigenvalues $\lambda_{k}$ being separated from the discrete eigenlevel $\bar{\lambda}$ by the gap $\Delta \lambda$, which depends on the peculiarities of the potential function $U(z)$. The obvious relation $\Delta \lambda=\lambda_{\min }-\bar{\lambda} \equiv \lambda_{\min }$ holds, where by $\lambda_{\min }$ we denote the bottom of the continuous spectrum, namely, one has that $\lambda_{\min } \leq \lambda_{k}$ where $\lambda_{\min }=\min \left\{R^{\prime}\left(u_{1}\right), R^{\prime}\left(u_{3}\right)\right\}$ (see Fig. 2). Finally, we notice that the separate eigenlevel $\bar{\lambda}$ being related to the translational mode is the lowest (minimal) eigenvalue of the spectrum $\left\{\lambda_{\alpha}\right\}$, namely, the rigorous equality $\bar{\lambda} \equiv \min \left\{\lambda_{\alpha}\right\}=0$ holds, in any case of the front solution $u_{0}(z)$ joining two steady states of the bistable system (e.g., see Refs. [18]). Referring to the particular case of the pseudolinear rate function we thus get that $\Delta \lambda=\lambda_{\min }$ where $\lambda_{\min }=\min \left\{\alpha_{1}, \alpha_{3}\right\}$. Finally, we notice that the slowly developing processes that are characterized by the low relaxation rates $\tau_{\alpha}^{-1}$ play a major role, in considering the retardation effects discussed. Consequently, in evaluating the characteristic (largest) relaxation time $\tau_{\mathrm{R}}$ of the "pseudolinear" system being only slightly deviated from its stationary state $u_{0}(z)$ we get that

$$
\tau_{\mathrm{R}}=\max \left\{\alpha_{1}^{-1}, \alpha_{3}^{-1}\right\} .
$$

From Eq. (14) it follows that the characteristic time duration $\tau_{\mathrm{R}}$ is a function of the outer slope coefficients of the rate function, $\alpha_{1}$ and $\alpha_{3}$, and is not sensitive to the inner $\alpha_{2}$. Furthermore, the characteristic relaxation rates of the system, $\tau_{\mathrm{R}}^{-1}$, derivable by the symmetrical and asymmetrical rate functions satisfying the relations $\alpha_{1}=\alpha_{3}$ and $\alpha_{1} \neq \alpha_{3}$ differ. By tuning the outer slope coefficient, either $\alpha_{1}$ or $\alpha_{3}$, one lengthens or shortens the time duration $\tau_{\mathrm{R}}$, as a consequence, one arrives at the different rates of the transient processes in a weakly perturbed system being slightly deviated from its stationary state $u_{0}(z)$.

\subsection{Bistable front under rectangular ac forcing: retarded speed functions}

The above given estimate (14) of the characteristic parameter $\tau_{\mathrm{R}}$ is very approximate. Let us turn to the more general case of the system being largely deviated from its stationary state $u_{0}(z)$.

As already noted, in considering the retarded accelerations of the ac driven front, more exactly, the lag time between the ac drive and the instantaneous velocity of BFs we shall deal with the step-like forcing function (9). The rectangular forcing (9) is extremely fast; both parameters discussed, $\tau_{\mathrm{R}}$ and $\tau_{D}$, that characterize the rate of the temporal relaxation of the transient processes in system and the time lags, respectively, coincide. The numerically found $s-t$ dependences that describe the response of BF to the step-like force (9) are shown by the solid curves 1, 2, and 3 in Fig. 3; the particular case of the symmetrical rate function is presented by curve 1 , for comparison. One can see that the presented $s-t$ dependences being derived at the different slope coefficients $\alpha_{3}$ are differently sloped, i.e., they are characterized by the different lags $\tau_{D}$. We notice that the presented dependences are in a reasonable agreement with relation (14) being derived within the perturbation theory. Indeed, the parameter values $\tau_{\mathrm{R}}$ that are related to the separate curves in Fig. 3 are as follows (see the figure caption): $\tau_{\mathrm{R}}=1$ (curves 1,2 ) and $\tau_{\mathrm{R}}=5$ (curve 3 ), whence it follows that $\tau_{\mathrm{R} 1}=\tau_{\mathrm{R} 2}<\tau_{\mathrm{R} 3}$ where the subscripts 1,2 , and 3 refer to the separate curves. As discussed, in the considered case of the rectangular forcing both parameters $\tau_{\mathrm{R}}$ and $\tau_{D}$ coincide, thus, we get that $\tau_{D 3}>\tau_{D 1}, \tau_{D 2}$. One can see that the presented $s-t$ dependences are in a qualitatively good agreement with relation $\tau_{D 3}>\tau_{D 1}, \tau_{D 2}$. Indeed, both nearly equally sloped curves 1 and 2 being derived at the different values of the slope coefficient $\alpha_{3}$ satisfying the relations $\alpha_{3}=\alpha_{1}$ and $\alpha_{3}>\alpha_{1}$, respectively, are related to the same value of the parameter $\tau_{\mathrm{R}}$, i.e., the approximate equality $\tau_{D 1} \approx \tau_{D 2}$ holds. By contrast, curve 3 being derived at the lesser slope coefficient $\alpha_{3}$ satisfying the relation $\alpha_{3}<\alpha_{1}$, thus, being related to the lower relaxation rate $\tau_{\mathrm{R}}^{-1}$ is more gently sloped, in accord with the relation $\tau_{D 3}>\tau_{D 1}, \tau_{D 2}$.

To be more specific we notice that the temporal behavior of the retarded speed functions $s(t)$ derivable by the rectangular forcing is exponential with a good accuracy of few percent, as follows from our direct calculations (see also Ref. [19]). Namely, the actual (numerically found) 


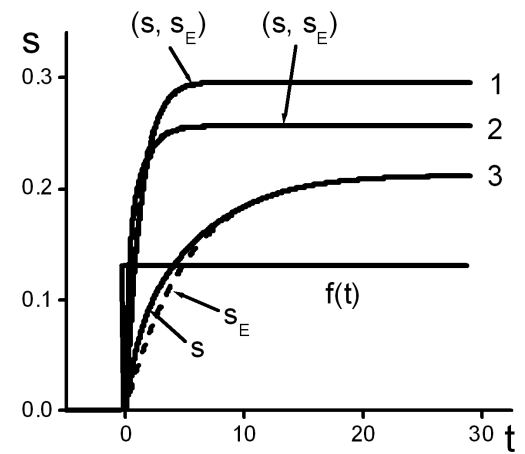

Fig. 3. The temporal relaxation of the moment velocity of the dragged front being under the action of the step-like force (shown by the rectangular curve). The solid curves show the actual $s-t$ dependences being derived by the numerical simulation of the governing Eq. (1), and the analytic approximations $s_{\mathrm{E}}(t)$ are shown by the dashed curves. The parameter values are: $\Delta R=1, \alpha_{1}=\alpha_{2}=1, h_{\mathrm{R}}=h_{0} ; F_{0}=-0.5 f_{\mathrm{Mx}}, t_{0}=0 ;$ (curve 1) $\alpha_{3}=1, \tau_{\mathrm{R}}=1.0, \tau_{S}=1.23, s_{\infty}=0.30$; (curve 2) $\alpha_{3}=5.0, \tau_{\mathrm{R}}=1.0, \tau_{S}=0.93, s_{\infty}=0.26$; (curve 3) $\alpha_{3}=0.2, \tau_{\mathrm{R}}=5.0, \tau_{S}=5.04, s_{\infty}=0.21$.

$s-t$ dependences are describable adequately enough by use of the following expression:

$$
\begin{aligned}
& s_{\mathrm{E}}\left(t-t_{0}\right)-s_{0} \\
& \quad= \begin{cases}0, & t<t_{0}, \\
\left(s_{\infty}-s_{0}\right)\left[1-\exp \left(-\left(t-t_{0}\right) / \tau_{S}\right)\right], & t>t_{0},\end{cases}
\end{aligned}
$$

where by $s_{0}$ and $s_{\infty}$ we denote the initial and asymptotic values of the speed function $s(t)$, respectively. More specifically, one has that $s\left(t<t_{0}\right)=s_{0}$ and $s(t \rightarrow \infty)$ $\rightarrow s_{\infty}$. The empirical parameter $\tau_{S}$ (below referred to as the "speed relaxation" time) characterizes the time duration of the transient behavior (temporal relaxation) of the step-like speed function; its inverse, $\tau_{S}^{-1}$, indicates the steepness of $s-t$ dependence. From Eq. (23) it follows that

$$
\frac{s_{\infty}-s_{\mathrm{E}}\left(T_{S}\right)}{s_{\infty}-s_{0}}=\delta_{S}, \quad \delta_{S}\left(T_{S}\right)=\mathrm{e}^{-\beta},
$$

where the introduced quantity, $T_{S}=t-t_{0}$, denotes the time separation between the time moment $t$ and the initial moment $t_{0}$, at which the step-like force was switched on, and the parameter $\beta$ is defined as follows, $\beta=T_{S} / \tau_{S}$. The characteristic time duration $\tau_{S}$ is derivable by the direct substitution of the auxiliary parameters $s_{0}$ and $s_{\infty}$, being derived by the numerical solution of the governing Eq. (1), into Eq. (15a). By properly choosing the empirical parameter $\delta_{S}$ one can find the parameter value $\tau_{S}$ with a needed accuracy. In considering the time lags discussed we used Eq. (15a). Clearly, the required parameter value $s_{\infty}$ derivable by numerically simulating the drift motion of the ac driven BF is very approximate; strictly speaking, the asymptotic value $s_{\infty}$ is numerically not achievable. In view of this, to enhance the accuracy of the calculations we used the required asymptotic values $s_{\infty}$ being derived by use of the "instantaneous" speed functions $s_{\mathrm{A}}(t)$. The speed function $s_{\mathrm{A}}(t)$ instantly follows the step-like rectangular forcing, namely, the one has that $s_{\infty}=s_{\mathrm{A}}\left(t>t_{0}\right)$.

The actual speed functions $s(t)$ and their emulations $s_{\mathrm{E}}(t)$ are presented in Fig. 3, for comparison; these are shown by the solid and dashed curves, respectively. One can see that a close proximity between the appropriate $s-t$ and $s_{\mathrm{E}}-t$ dependences being derived at the same values of the outer slope coefficient $\alpha_{3}$ occurred. For large $t$ 's satisfying the relation $t-t_{0} \gg \tau_{\mathrm{R}}$ both dependences discussed practically coincide. They deviate somewhat from each other only in the interval of the initial time moments satisfying the relation $t-t_{0}<\tau_{\mathrm{R}}$. Such an exponential behavior of the retarded speed functions $s(t)$ may be attributed to the piecewise-linear character of the rate function $R(u)$.

In continuing the discussion of the retarded speed functions we notice that both parameters discussed, the speed relaxation time $\tau_{S}$ obtainable empirically and the characteristic time duration $\tau_{\mathrm{R}}$ derivable analytically, by use of the perturbation theory are in a reasonable agreement with each other, as follows from our direct calculations. A close proximity of both parameters discussed, $\tau_{S}$ and $\tau_{\mathrm{R}}$, is demonstrated by two separate families of $\tau_{S}-\alpha_{3}^{-1}$ and $\tau_{S}-\alpha_{2}^{-1}$ characteristics that are shown by the solid and dashed curves, respectively, in Fig. 4. The family of the solid curves labeled by $1 \mathrm{a}, 2 \mathrm{a}$, and $3 \mathrm{a}$ in the figure show $\tau_{S}-\alpha_{3}^{-1}$ dependences being derived at the different outer slope coefficients $\alpha_{1}$ of the rate function; the inner slope coefficient $\alpha_{2}$ was kept fixed. One can see that the presented $\tau_{S}-\alpha_{3}^{-1}$ dependences agree both qualitatively and quantitatively well enough with relation (14) being derived within the perturbation theory. Indeed, from Eq. (14) it follows that the character of the discussed $\tau_{\mathrm{R}}-\alpha_{3}^{-1}$ dependences is quite different within two separate intervals of the "variable" $\alpha_{3}^{-1}$, being defined by the following relations, $\alpha_{3}^{-1} \in\left(0, \alpha_{1}^{-1}\right]$ and $\alpha_{3}^{-1} \in\left[\alpha_{1}^{-1}, \infty\right)$, respectively. More specifically, one has that (i) $\tau_{\mathrm{R}}=\alpha_{1}^{-1}=$ const if $\alpha_{3}^{-1}<\alpha_{1}^{-1}$, and (ii) $\tau_{\mathrm{R}}=\alpha_{3}^{-1}$ if $\alpha_{3}^{-1}>\alpha_{1}^{-1}$. The "critical" (break) points of the considered $\tau_{\mathrm{R}}-\alpha_{3}^{-1}$ dependences, separating both intervals discussed, are located at $\alpha_{3}^{-1}=\alpha_{1}^{-1}$; the break points are pointed by the vertical arrow-lines in Fig. 4, and the straight dashed line $A$ shows the linear $\tau_{\mathrm{R}}-\alpha_{3}^{-1}$ dependence (ii). One can see that in the limit of the large $\alpha_{3}^{-1}$ 's satisfying the relation $\alpha_{3}^{-1} \gg \alpha_{1}^{-1}$ the numerically found $\tau_{S}-\alpha_{3}^{-1}$ characteristics closely approach the linear dependence $\tau_{S}=\alpha_{3}^{-1}$ shown by the dashed line $A$ in the figure, in a good agreement with relation (ii). By contrast, in the interval of the small values $\alpha_{3}^{-1}$ satisfying the relation $\alpha_{3}^{-1} \ll \alpha_{1}^{-1}$ the empirical parameter $\tau_{S}$ tends to some fixed value, $\tau_{S} \approx$ const, in accord with relation (i) (see curves 1a, 2a, and $3 \mathrm{a}$ in the figure). Both dependences $\tau_{S}-\alpha_{3}^{-1}$ and $\tau_{\mathrm{R}}-\alpha_{3}^{-1}$ deviate some- 


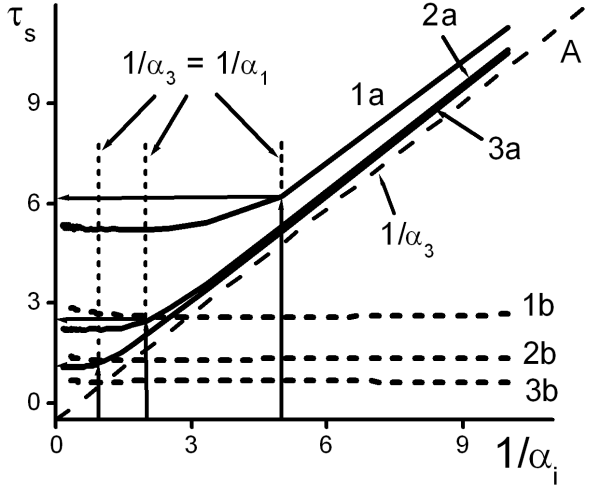

Fig. 4. The speed relaxation time $\tau_{S}$ versus the inverse slope coefficients $\alpha_{3}^{-1}$ and $\alpha_{2}^{-1}$ of the rate function; two separate families of $\tau_{S}-\alpha_{3}^{-1}$ and $\tau_{S}-\alpha_{2}^{-1}$ dependences being derived at the different values of the outer slope coefficients, $\alpha_{1}$ and $\alpha_{3}$, respectively, are shown by the solid and dashed curves labeled by a and b, respectively. The vertical arrows, being located at the break points $\alpha_{3}^{-1}=\alpha_{1}^{-1}$ of $\tau_{\mathrm{R}}-\alpha_{3}^{-1}$ characteristic, on the abscissa axis, separate two different intervals $\alpha_{3}^{-1}<\alpha_{1}^{-1}$ and $\alpha_{3}^{-1}>\alpha_{1}^{-1}$ of the variable $\alpha_{3}$, that are characterized by the different $\tau_{\mathrm{R}}-\alpha_{3}^{-1}$ dependences. Namely, one has that (a) $\tau_{\mathrm{R}}=\alpha_{1}^{-1}$ if $\alpha_{3}^{-1}<\alpha_{1}^{-1}$, and (b) $\tau_{\mathrm{R}}=\alpha_{3}^{-1}$ if $\alpha_{3}^{-1}>\alpha_{1}^{-1}$ (see Eq. (14)). The horizontal arrows point the parameter values $\tau_{S}\left(\alpha_{3}^{-1}\right)$ taken at the break points, $\alpha_{3}^{-1}=\alpha_{1}^{-1}$. The straight dashed line labeled by A shows the linear dependence, $\tau_{S}=\alpha_{3}^{-1}$. The parameter values are: $\Delta R=1, h_{\mathrm{R}}=1.2 h_{0} ; F_{0}=-2 f_{\mathrm{M} x} / 3 ;$ (curves 1a, $2 \mathrm{a}$, and 3a) $\alpha_{2}=1$ and $\alpha_{1}=0.2,0.5,1.0$, respectively; curves $(1 \mathrm{~b}, 2 \mathrm{~b}$, and $3 \mathrm{~b}) \alpha_{1}=\alpha_{3}=0.5,1.0,2.0$, respectively. The characteristic parameters $\tau_{\mathrm{R}}$ being related to the separate curves $1 \mathrm{~b}, 2 \mathrm{~b}$, and $3 \mathrm{~b}$ are as follows: $\tau_{\mathrm{R}}=2.0 ; 1.0 ; 0.5$, respectively.

what from each other in the vicinity of the break points being located at $\alpha_{3}^{-1}=\alpha_{1}^{-1}$. For instance, the minimal (lowest) values $\tau_{\min }$ of the empirical parameter $\tau_{S}$ being related to the separate curves shown Fig. 4 are as follows: $\tau_{\text {min }} \approx 5.2(1 \mathrm{a}), 2.2(2 \mathrm{a}), 1.1$ (3a) where the notations in the round brackets refer to the separate curves. On the other hand, the minimal values of the characteristic relaxation time $\tau_{\mathrm{R}}$ being related to the separate curves $1 \mathrm{a}$, 2a, and 3a are as follows: $\tau_{\mathrm{R}}=5.0(1 \mathrm{a}), 2.0(2 \mathrm{a}), 1.0(3 \mathrm{a})$ (see the figure caption), thus, a close proximity between both discussed parameters occurred.

Referring to the $\tau_{S}-\alpha_{2}^{-1}$ characteristics that describe the dependence of the empirical parameter $\tau_{S}$ versus the inner slope coefficient we notice that a close proximity between both $\tau_{S}-\alpha_{2}^{-1}$ and $\tau_{\mathrm{R}}-\alpha_{2}^{-1}$ dependences has also occurred (see the dashed curves in Fig. 4). One can see that the presented $\tau_{S}-\alpha_{2}^{-1}$ dependences that are derived at the different values of the outer slope coefficients $\alpha_{1,3}$ are flattened; the characteristic time duration $\tau_{S}$ is practically independent of the inner slope coefficient $\alpha_{2}$, in a good agreement with relation (14). Furthermore, both discussed parameters $\tau_{S}$ and $\tau_{\mathrm{R}}$ are close to each other, namely, one has that i) $\tau_{S} \approx 2.9(1 \mathrm{~b}), 1.4(2 \mathrm{~b}), 0.7(3 \mathrm{~b})$, and ii) $\tau_{\mathrm{R}} \approx 2.0(1 \mathrm{~b}), 1.0(2 \mathrm{~b}), 0.5(3 \mathrm{~b})$ where, as before, the notations in the braces refer to the separate curves.

Thus, we conclude that a close proximity between both $\tau_{S}-\alpha_{i}$ and $\tau_{\mathrm{R}}-\alpha_{i}$ dependences was occurred. We notice that the strength of the rectangular forcing that was used in the derivation of the considered $\tau_{S}-\alpha_{i}$ dependences was taken large enough, namely, one had that $\left|F_{0}\right| \approx$ $0.7 f_{\mathrm{M} x}$ (see the figure caption). By contrast, the relation (14) holds in the case of the weak forcing only.

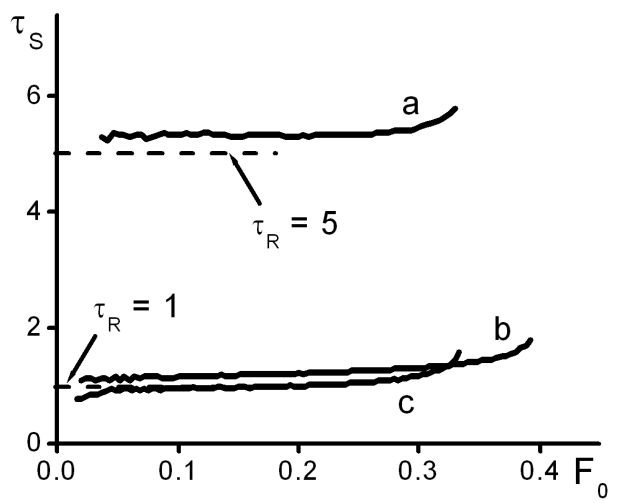

Fig. 5. The characteristic time duration $\tau_{S}$ versus the strength $F_{0}$ of the rectangular forcing. The parameter values are: $\Delta R=1, h_{\mathrm{R}}=2 h_{0} / 3, \alpha_{1}=\alpha_{2}=1$; (curve a) $\alpha_{3}=0.2, h_{\mathrm{R}} \approx 1.55, s_{0}=-0.09 ;$ (curve b) $\alpha_{3}=1.0, h_{\mathrm{R}}=2 / 3, s_{0}=-0.11 ;$ (curve c) $\alpha_{3}=5.0$, $h_{\mathrm{R}} \approx 0.52, s_{0}=-0.12$. The characteristic parameters $\tau_{\mathrm{R}}$ being related to the separate curves $\mathrm{a}, \mathrm{b}$, and $\mathrm{c}$ are as follows: $\tau_{\mathrm{R}}=5.0,1.0$, and 1.0 , respectively (shown by the dashed horizontal lines in the figure).

Evidently, the characteristic time duration $\tau_{S}$ should be a function of the strength of the ac forcing, $F_{0}$. We notice in advance that the empirical parameter $\tau_{S}$ has only a weak dependence on the strength of the rectangular forcing; the numerically found $\tau_{S}-F_{0}$ dependences derivable by the flexible rate function (5) are gently sloped, as follows from our direct calculations. The typical $\tau_{S}-F_{0}$ characteristics are presented by solid curves a, b, and c in Fig. 5; the dashed horizontal lines point the corresponding values of the parameter $\tau_{R}$. One can see that the presented curves are flattened everywhere, apart from the narrow interval of the extremely large $F_{0}$ 's being in a close vicinity of the critical point, $F_{0}=f_{M x}$, above which the global stability of the ac BFs breaks down. We notice that the parameter values $\tau_{S}\left(F_{0}\right)$ that are taken within the interval of the small amplitudes $F_{0}$ satisfying the relation $F_{0} \ll f_{\mathrm{M} x}$ are very close to those derivable within the perturbation theory, namely, the approximate equality holds, $\tau_{S}\left(F_{0}\right) \approx \tau_{\mathrm{R}}$ if $F_{0} \ll f_{\mathrm{M} x}$. Referring to the separate curves shown in Fig. 5 we get that $\tau_{S} \approx 5.3$ (a), 1.1 (b), and 0.8 (c) if $F_{0}<0.1$, where the notations in the braces refer to separate curves. Quite similarly, from Eq. (14) it follows that $\tau_{\mathrm{R}}=5.0$ (a), 1.0 (b), and 1.0 (c).

Summarizing we conclude that (a) the transient behavior of the step-like speed functions $s(t)$ that describe the 
temporal evolution of the instantaneous velocity of the ac driven BFs under the rectangular forcing is exponential, (b) the characteristic exponent of the retarded speed functions, $\tau_{S}^{-1}$, that describes both the rate of the temporal relaxation of the speed functions and the time lags between the instantaneous velocity of the ac driven $\mathrm{BF}$ and the driving force is a function of the outer slope coefficients of rate function, $\alpha_{1,3}$, and is not sensitive to the inner $\alpha_{2},(\mathrm{c})$ the characteristic time duration $\tau_{S}$ has only weak dependence on the strength of the applied forcing, (d) the temporal behavior of the retarded speed functions $s(t)$ being derived with an arbitrary strong forcing is describable adequately enough by use of the perturbation theory, in the most general case of the piecewise linear rate function.

Obviously, the retardation effects discussed will take place also in the case of the periodic oscillatory zeromean ac forces being capable to induce the directed net motion of the ac driven BF. The occurrence of the time lags between the oscillatory force and the instantaneous velocity of the periodically forced BFs may significantly influence on the spurious drift of BFs. Let us turn to the rectified oscillatory motion of the ac driven BFs derivable by the asymmetrical rate functions.

\section{Front dynamics with time lags}

In considering the ratchet-like transport of BFs we shall deal with the single-harmonic forcing function of the highest temporal symmetry. The scaled forcing functions $f^{*}(t)$ being defined by the relation $f^{*}(t):=f(t) / \Delta R$ will be used. The average characteristics of the spurious drift of BFs derivable by the self-similar rate functions $R(u ; C)$ are not sensitive to the height $\Delta R$ of the rate function if both scaled functions $f^{*}(t)$ and $s(t)$ are used (see Refs. $[8,10,12])$.

In the previous section we have demonstrated that both relaxation rates $\tau_{\mathrm{R}}^{-1}$ and $\tau_{S}^{-1}$ derivable in two different cases of the symmetrical and asymmetrical rate functions notably differ. Broadly speaking, the parameters discussed are increasing functions of the outer slope coefficients of the rate function. Thus, one may expect that the lag time between the oscillatory speed function $s(t)$ of the periodically forced BF and the ac force will be decreasing function of the outer slope coefficients. Our numerical simulations confirm this prediction. The oscillatory speed functions $s(t)$ that are derived at the different slope coefficients $\alpha_{3}$ are presented by the solid curves in Fig. 6; the dashed curves show the "instantaneous" speed functions $s_{\mathrm{A}}(t)$. As expected, the actual speed functions $s(t)$ become more and more flattened with the decreasing slope coefficient $\alpha_{3}$ (compare the solid curves shown in the figure). Both the magnitude, $\Delta s=s_{\mathrm{M}}-s_{\mathrm{m}}$, of the oscillatory function $s(t)$ and the lag time between both $s-t$ and $s_{\mathrm{A}}-t$ dependences increase with the decreasing slope coefficient $\alpha_{3}$, in accord with relation (14); as before, by the subscripts $\mathrm{M}$ and $\mathrm{m}$ we denote "Maximal" and "minimal", respectively. The increasing time lags between both oscillatory functions $s(t)$ and $s_{\mathrm{A}}(t)$ are schematically depicted by the straight inclined lines (a) and (b) denoting the average "slopes" of the considered $s_{\mathrm{A}}-t$ and $s-t$ dependences (the straight inclined line indicating the average "slope" of the oscillatory function joins the adjacent extremes of the oscillatory function). One can see that the relative slope between both inclined lines (a) and (b) increases with the decreasing slope coefficient $\alpha_{3}$ (compare the separate families of the curves labeled (A), (B) and (C) in the figure). Notice that the magnitude of the actual (retarded) speed function, $\Delta s$, becomes notably reduced even at the moderately low frequencies satisfying the relation $\omega \simeq \tau_{\mathrm{R}}^{-1}$. Referring to the particular case labeled by (B) in the figure we get that $\Delta s / \Delta s_{\mathrm{A}} \approx 0.3$ where the quantity, $\Delta s_{\mathrm{A}}=s_{\mathrm{AM}}-s_{\mathrm{Am}}$, denotes the magnitude of the "instantaneous" oscillatory function $s_{\mathrm{A}}(t)$ that ignores the time lags discussed (notice, both parameters $\tau_{\mathrm{R}}^{-1}$ and $\omega$ that characterize the "rate" of the system and the oscillatory force, respectively, are of the same order in magnitude in this case, namely, one has that $\omega=6 \tau_{\mathrm{R}}^{-1}$; see the figure caption). The magnitude $\Delta s$ of the actual speed function $s(t)$ becomes notably reduced, namely, the inequality $\Delta s \ll \Delta s_{\mathrm{A}}$ holds if the criterion of the rapid driving, $\omega \tau_{\mathrm{R}} \gg 1$, is satisfied, as follows from our direct calculations. In closing the discussion of the oscillatory speed functions we conclude that the temporal behavior of the retarded speed functions $s(t)$ is in line with the previous results being derived with the rectangular forcing. Let us turn to the spurious drift of BFs.

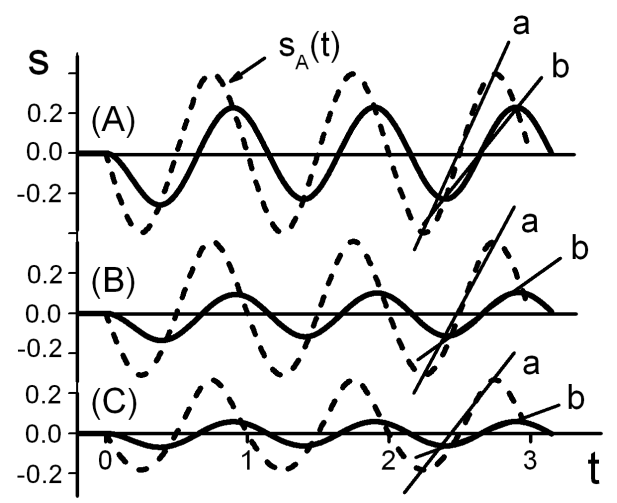

Fig. 6. The retarded speed functions $s(t)$ being derived at the different values of the outer slope coefficient $\alpha_{3}$ (shown by the solid curves). The dashed curves show the "instantaneous" speed functions $s_{\mathrm{A}}(t)$ that ignore the delays. The average slopes of both speed functions $s_{\mathrm{A}}(t)$ and $s(t)$ are depicted by the straight inclined lines (a) and (b), respectively. Notice that the relative slope (angle) between both lines (a) and (b) increases with the decreasing slope coefficient $\alpha_{3}$. The parameter values are: $f_{0}^{*}=0.6 f_{\mathrm{M} x}^{*}, T=1 ; \alpha_{1}=5, \alpha_{2}=1, h_{\mathrm{R}}=h_{0}$, $s_{0}=0 ;$ (curves A) $T=5.0 \tau_{\mathrm{R}}, \alpha_{3}=5.0, h_{\mathrm{R}}=1.00$; (curves B) $T=\tau_{\mathrm{R}}, \alpha_{3}=1, h_{\mathrm{R}}=1.29$; (curves C) $T=$ $0.2 \tau_{\mathrm{R}}, \alpha_{3}=0.2, h_{\mathrm{R}}=2.24$. The forcing amplitudes $f_{0}^{*}$ being related to the separate pairs of the curves labeled by $A, B$, and $C$ are as follows: $f_{0}^{*} \approx 0.30,0.26$, and 0.19 , respectively. 


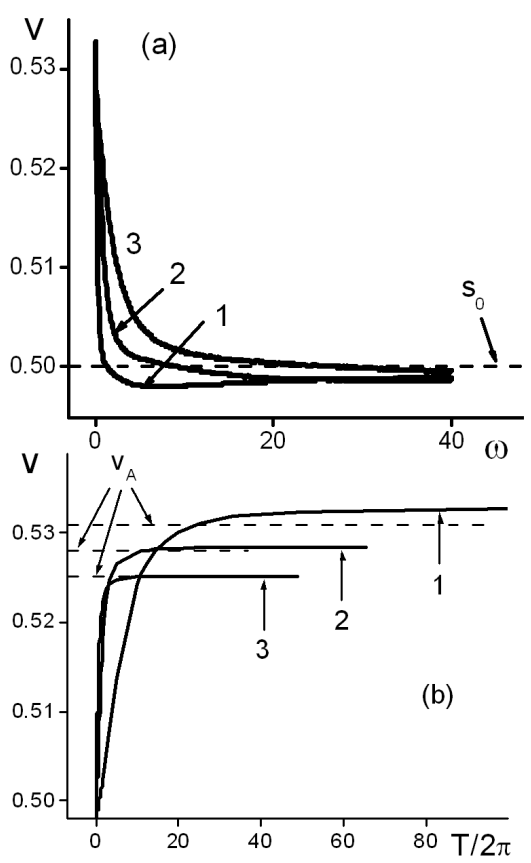

Fig. 7. (a) The mean drift velocity of the ac driven $\mathrm{BF}$ versus the frequency of the harmonic force. The initial velocity of $\mathrm{BF}$ (shown by the dashed line) was taken fixed, namely, $s_{0}=0.5$. The parameter values are: $f_{0}^{*}=0.9 f_{\mathrm{M} x}^{*} ; \alpha_{1}=5, \alpha_{2}=1 ;$ (curve 1$) \alpha_{3}=0.2$, $h_{\mathrm{R}} \approx 9.6 h_{0}\left(h_{0} \approx 2.24\right), f_{0}^{*} \approx 0.04$; (curve 2$) \alpha_{3}=1.0$, $h_{\mathrm{R}} \approx 6.9 h_{0}\left(h_{0} \approx 1.29\right), f_{0}^{*} \approx 0.09 ;$ (curve 3$) \alpha_{3}=5.0$, $h_{\mathrm{R}} \approx 6.3 h_{0}\left(h_{0}=1.00\right), f_{0}^{*} \approx 0.12$. The characteristic time durations $\tau_{\mathrm{R}}$ being related to the separate curves (1), (2), and (3) are as follows: $\tau_{\mathrm{R}}=5.0,1.0$, and 0.2 , respectively. Notice that the steepness of the given $v-\omega$ dependences increases with the decreasing slope coefficient $\alpha_{3}$. In the range of the high frequencies satisfying the relation $\omega \tau_{\mathrm{R}} \gg 1$ the mean drift velocity of $\mathrm{BF}$ is very close to that of the free $\mathrm{BF}, s_{0}$, i.e., the spurious drift of BF practically disappears. (b) The same as in Fig. 7a, but the dependence of the mean drift velocity of $\mathrm{BF}$ versus the period of the ac force is presented. Both the denotations and the parameter values are the same as in part (a). The dashed horizontal lines show the mean drift velocities $v_{\mathrm{A}}$ being derived by the speed equation (8); these are as follows: (curve 1) $v_{\mathrm{A}} \approx 0.531 ;$ (curve 2 ) $v_{\mathrm{A}} \approx 0.528 ;$ (curve 3 ) $v_{\mathrm{A}} \approx 0.524$. The actual drift velocities $v$, when taken at the fixed period, $T=100$, which satisfies the relation $T \gg \tau_{\mathrm{R}}$, are as follows: (curve 1) $v \approx 0.533$; (curve 2 ) $v \approx 0.528$; (curve 3) $v \approx 0.525$.

The influence of the retardation effects discussed on the ratchet-like transport of BFs is demonstrated by $v-\omega$ dependences shown by the solid curves 1, 2, and 3 in Fig. 7a. The average characteristics that are derived with the asymmetrical rate functions are shown by curves 1 and 2 , and the particular case of the symmetrical rate function is presented by curve 3 .

As expected, the presented $v-\omega$ dependences that are related to the lesser values of the outer slope coefficient $\alpha_{3}$, thus, being related to the lower characteristic rates $\tau_{\mathrm{R}}^{-1}$ and the larger lags $\tau_{D}$ are more steeply sloped, in accord with relation (14). To be more specific we notice that the relaxation rates $\tau_{\mathrm{R}}^{-1}$ being related to the separate curves 1,2 , and 3 are as follows: $\tau_{\mathrm{R}}^{-1}=0.2,1.0$, and 5.0 , respectively. It is necessary to note that the "acceleration factor" of the ac driven BF, $\rho(\omega)=v(\omega) / s_{0}$, which characterizes the "efficiency" of the ac driver, becomes notably reduced, i.e., the drift velocity $v$ of the ac driven $\mathrm{BF}$ becomes very close to that of free, unperturbed BF, $s_{0}$, even at "moderately low" frequencies $\omega$ satisfying the relation $\omega \tau_{\mathrm{R}} \simeq 1$. More specifically, the acceleration factors, $\rho_{\mathrm{R}} \equiv \rho\left(\omega=\tau_{\mathrm{R}}^{-1}\right)$, being related to the separate curves 1, 2, and 3 shown in Fig. 7a are as follows: 1.03, 1.02 , and 1.01 , respectively.

In order to reveal the peculiarities of the ratchet-like shuttling of BFs in the low-frequency range of the oscillatory force we present the "reciprocal" $v-T$ dependences that describe the dependence of the mean drift velocity $v$ versus the period $T$ of the forcing. These are shown in Fig. 7b; both the notations and the parameters used are the same as in Fig. 7a. As expected, the average $v-T$ characteristics are decreasingly sloped with the increasing period of the forcing. The mean drift velocity $v$ of the ac driven BF becomes very close to that of the quasi-stationary driven $\mathrm{BF}, v_{\mathrm{A}}$, if the period $T$ of the ac force significantly exceeds the characteristic relaxation time of the system, $\tau_{\mathrm{R}}$ (the drift velocities $v_{\mathrm{A}}$ are pointed by the dashed horizontal lines in the figure). This result harmonizes well with the previous findings (see Fig. 6); the retardation effects become notably reduced, i.e., the lag time $\tau_{D}$ practically vanishes if the criterion of the slow driving $T \gg \tau_{\mathrm{R}}$ is satisfied.

In closing the discussion of the spurious drift of the "retarded" BFs let us touch briefly on some peculiarities of the reversal behavior of the directed net motion of the ac driven BFs derivable by the asymmetrical rate functions. As previously mentioned, the average $v-f_{0}^{*}$ characteristics of the ratchet-like transport of BFs that are derived by use of the symmetrical and asymmetrical rate functions radically differ. Our previous calculations being performed by use of the asymmetrical rate functions, within the adiabatic approximation discussed, demonstrated that depending on the initial velocity of the free, unperturbed $\mathrm{BF}, s_{0}$, the ac driven BF being subjected to the time-symmetric oscillatory force of zero-time average exhibited the reversal of the unidirectional net motion, as a function of the driving amplitude $f_{0}$ (see Ref. [8]). The reversal implies that the average $v-f_{0}^{*}$ characteristic of the ac driven BF has at least one null (zero-point). As already mentioned, the governing Eq. (1) is analytically not solvable; the rigorous criteria under which the reversals discussed exist (occur, vanish) are lacking. The approximate criteria, more exactly, the necessary, but insufficient conditions for the existence of the reversals discussed are obtainable by considering the spurious drift of BFs under "slow" square-wave ac forcing satisfying the relation $T \rightarrow \infty$ (see Ref. [8]). The approximate criteria encompassing both cases of the backward- and forward-running BFs satisfying the relations $s_{0}<0$ and $s_{0}>0$, respec- 
tively, read: (A) $h_{\mathrm{R}} \in\left(1, h_{0}\right)$ if $\alpha_{1}>\alpha_{3}$ (the particular case, $\left.s_{0}<0\right)$; (B) $h_{\mathrm{R}} \in\left(h_{0}, 1\right)$ if $\alpha_{1}<\alpha_{3}$ (the other case, $\left.s_{0}>0\right)$; the boundary conditions $u_{0}(z \rightarrow \mp \infty) \rightarrow u_{1,3}$ discussed above have been used in the derivation of these relations. We stress that the presented criteria (A) and (B) are the necessary, but not sufficient conditions for the existence of the reversals discussed. In any case, the reversal disappears, namely, the progressive (accelerated) dc motion of the ac driven BF takes place if both criteria (A) and (B) are broken. The adiabatic approximation being used in the derivation of the presented relations (A) and (B) ignores the time lags between the instantaneous velocity of the ac driven $\mathrm{BF}$ and the ac forcing. It is not clear enough whether and how strongly the retardation effects discussed are capable to influence on the reversal behavior of the unidirectional net motion of the ac driven BFs.

The numerically found $v-f_{0}^{*}$ dependences encompassing both cases of the progressive and regressive dc motion of BFs are presented by the solid curves in Figs. 8A and $\mathrm{B}$, respectively. The dashed curves labeled by $v_{\mathrm{A}}$ show the average $v_{\mathrm{A}}-f_{0}^{*}$ characteristics being derived by the speed Eq. (8). One can see that the numerically found $v-f_{0}^{*}$ dependences that describe the particular case of the slow oscillatory force satisfying the relation $T \gg \tau_{\mathrm{R}}$ agree well with the appropriate $v_{\mathrm{A}}-f_{0}^{*}$ dependences being derived within the adiabatic approximation discussed (compare the solid and the dashed curves labeled by a and $v_{\mathrm{A}}$, respectively, in both figures). As expected, the steepness of the given $v-f_{0}^{*}$ characteristics decreases with the frequency of the ac force being increased; the spurious drift of BF becomes notably suppressed in the whole interval of the driving amplitudes $f_{0}$ if the period of the ac force becomes lesser if compared to the characteristic relaxation time of the system, namely, if the relation $T \leq \tau_{\mathrm{R}}$ is satisfied (see curves labeled by $\mathrm{c}$ in both figures). As noted, the reversals in the spurious drift of BFs are related to the nulls of $v-f_{0}^{*}$ characteristics. The particular case of the regressive-progressive dc motion exhibiting the reversal that satisfies the criterion $(\mathrm{A})$, is demonstrated by curve (a) in Fig. $8 \mathrm{~B}$.

The occurrence of the time lags in the oscillatory motion of BF leads to the shifting of the nulls of $v-f_{0}^{*}$ characteristic toward the larger values of the driving amplitude $f_{0}^{*}$; the higher strengths of the oscillatory force are required to achieve the reversal with the ac force of the higher frequency $\omega$. Curve (b) shows the "critical" situation: the ac driven front, which initially propagated at some fixed nonzero velocity $s_{0}$, experienced the stopping at the maximal amplitude, $f_{0}^{*}=f_{\mathrm{M} x}^{*}$, above which the global stability of BF breaks down. A further increase of the oscillatory frequency $\omega$ leads to the larger lags, as a consequence, the average $v-f_{0}^{*}$ characteristic becomes more and more flattened with the increasing frequency $\omega$, as a result, the reversal of the unidirectional net motion of $\mathrm{BF}$ disappears (see curve $\mathrm{c}$ in Fig. $8 \mathrm{~B}$ ). As noted, the rigorous criteria for the existing of the reversals discussed are lacking. The average $v-f_{0}^{*}$ charac-

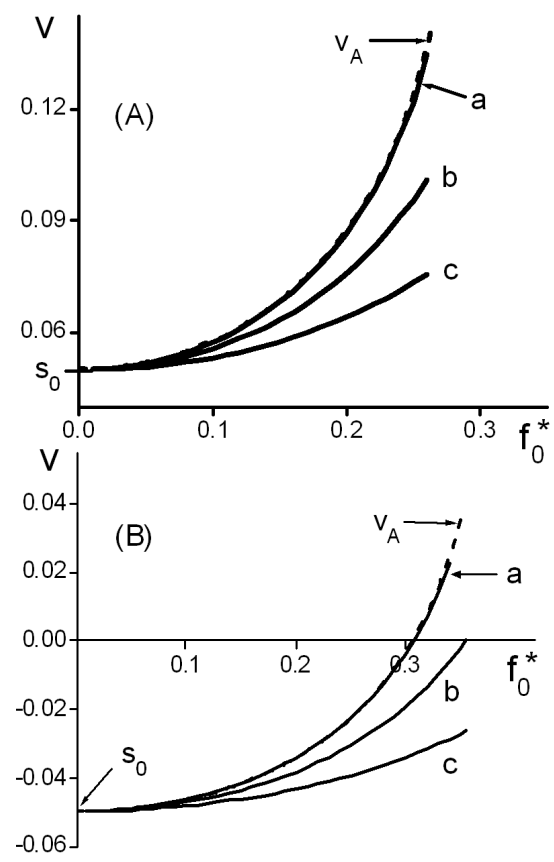

Fig. 8. (A) The mean drift velocity of the ac driven $\mathrm{BF}$ versus the amplitude of the harmonic force. The solid curves (a), (b), and (c) show the actual $v-f_{0}^{*}$ dependences being derived by the numerical solution of Eq. (1); the dashed curve labeled by $v_{\mathrm{A}}$ is obtained by the speed Eq. (8). The parameter values are: $\alpha_{1}=5$, $\alpha_{2}=1, \alpha_{3}=0.2 ; h_{0} \approx 2.24, h_{\mathrm{R}} \approx 2.78 ; s_{0}=0.05$, $\tau_{\mathrm{R}}=5.0 ;$ (curve a) $T / \tau_{\mathrm{R}}=60 ;$ (curve b) $T / \tau_{\mathrm{R}}=10$; (curve c) $T / \tau_{\mathrm{R}}=5$. The maximal drift velocities $v_{\mathrm{M} x}$ being derived at the maximal amplitude of the ac drive, $f_{0}^{*}=f_{\mathrm{M} x}^{*}$, are as follows: (curve a) $v_{\mathrm{M} x} \approx 0.13$; (curve b) $v_{\mathrm{M} x} \approx 0.10$; (curve c) $v_{\mathrm{M} x} \approx 0.08$. (B) The same as in part (A), but for the other case of the oppositely running BF satisfying the criterion of the reversal behavior (A). The parameter values are: $s_{0}=-0.05$; $\alpha_{1}=5, \alpha_{2}=1, \alpha_{3}=0.2 ; h_{0} \approx 2.24, h_{\mathrm{R}} \approx 1.81 ;$ $\tau_{\mathrm{R}}=5.0 ;$ (curve a) $T / \tau_{\mathrm{R}}=60 ;$ (curve b) $T / \tau_{\mathrm{R}}=10 ;$ (curve c) $T / \tau_{\mathrm{R}}=5$. The maximal drift velocities $v_{\mathrm{M} x}$ being related to the separate curves a, b, and $\mathrm{c}$ are as follows: $v_{\mathrm{M} x} \approx 0.042,0.000$, and -0.026 , respectively. Notice that depending on the frequency of the driving force, the bistable front, which initially propagated at the fixed velocity $s_{0}$, exhibited (i) the reversal (curve a), (ii) the stopping (curve b), (iii) regressive (decelerated) dc drift (curve c), as a function of the driving amplitude.

teristics become very flattened practically in the whole interval of the driving amplitudes $f_{0}^{*}$ if the lag time $\tau_{D}$ significantly exceeds the period of the forcing, namely, when the criterion of the fast driving $\omega \tau_{\mathrm{R}} \gg 1$ is satisfied, as follows from our direct calculations. Thus, we say that the reversals discussed will disappear "almost in the whole interval" of the initial propagation rates $s_{0}$ of the ac driven $\mathrm{BF}$ if the criterion of the rapid driving is satisfied.

\section{Summary}

The retardation effects (time lags) in dynamics of the ac driven "bistable" fronts (BFs) joining two states of the 
different stability in a bistable system of the reaction-diffusion type have been studied by use of the macroscopic kinetic equation of the reaction kinetics, within the piecewise linear model of the system, encompassing both cases of the symmetrical and asymmetrical rate functions. By numerically simulating the drift motion of the ac driven $\mathrm{BF}$ under the step-like ac forcing we have shown that $(\mathrm{A})$ the time-dependent speed functions that describe the temporal evolution of the instantaneous velocity of the ac driven $\mathrm{BF}$ follow the ac drive with some retardation (lag time), (B) the temporal behavior of the retarded (step-like) speed functions is exponential, in the most general case of the piecewise linear rate function of the flexible symmetry; the temporal evolution of the instantaneous velocity of the ac driven $\mathrm{BF}$ is describable quite adequately by use of the characteristic exponent $\gamma_{S}$ denoting the rate of the temporal relaxation of the retarded speed function; its inverse, $\tau_{S}=\gamma_{S}^{-1}$, describes the lag time $\tau_{D}$ between the instantaneous velocity of the ac driven $\mathrm{BF}$ and the rectangular forcing, (C) the lag time $\tau_{D}$ is a function of the outer slope coefficients of the rate function and is not sensitive to the inner; furthermore, it has only weak dependence on the strength of the applied forcing, (D) the time lags (retardation effects) in the front dynamics are describable by use of the perturbation theory, even in the case of the strong forcing.

Another aspect of the front dynamics being discussed in the present report is the influence of the retardation effects on the ratchet-like shuttling of BFs derivable by the asymmetrical rate functions. By considering the response of $\mathrm{BF}$ to the single-harmonic ac forcing we were able to show that the occurrence of the time lags in the oscillatory motion of the ac driven $\mathrm{BF}$ shrunk the spurious drift of BF; the spurious drift of BFs practically disappeared if the frequency of the oscillatory force acting of the front significantly exceeded the characteristic relaxation rate of the system. In addition, we have shown that the possibilities of controlling the directed net motion of the self-ordered fronts by the low- and high-frequency zero-mean ac forces radically differ: the occurrence of the time lags in the front dynamics leads to the vanishing of the reversals in the directed net motion of the ac driven BFs, being inherent in the case of the extremely slow (quasi-stationary) ac drive.

\section{References}

[1] F. Julicher, A. Adjari, J. Prost, Rev. Mod. Phys. 69, 1269 (1997); D. Abbott, Chaos 11, 526 (2001); P. Reimann, Phys. Rep. 361, 57 (2002); R. Mallik, P. Gross, Current Biol. 14, R971 (2004); P. Hanggi, F. Marchesoni, Rev. Mod. Phys. 81, 387 (2009).

[2] A V. Savin, G.P. Tsironis, Y. Zolotariuk, Phys. Rev. E 56, 2457 (1997); N. Quintero, A. Sanchez, Eur. Phys. J. B 6, 133 (1998); G. Costantini, F. Marchesoni, M. Borromeo, Phys. Rev. E $6 \mathbf{6 5}$ 051103 (2002); M. Salerno, Y. Zolotaryuk, Phys. Rev.
E 65, 056603 (2002); S. Flach, Y. Zolotaryuk, A.E. Morishnichenko, M.V. Fistul, Phys. Rev. Lett. 88, 184101 (2002); L. Morales-Molina, N.R. Quintero, F.G. Mertens, A. Sanchez, Phys. Rev. Lett. 91, 234102 (2003);N.R. Quintero, B. Sanchez-Rey, M. Salerno, Phys. Rev. E 72, 016610 (2005); L. Morales-Molina, N.R. Quintero, A. Sanchez, F.G. Mertens, Chaos 16, 013117 (2006).

[3] J. Armero, J.M. Sancho, J. Casademunt, A.M. Lacasta, L. Ramirez-Piscina, F. Sagues, Phys. Rev. Lett. 76, 3045 (1996); J. Armero, J. Casademunt, L. Ramirez-Piscina, J.M. Sancho, Phys. Rev. E 58, 5494 (1998); M.A. Santos, J.M. Sancho, Phys. Rev. E 64, 016129 (2001); Ch.R. Doering, C. Muller, P. Smereka, Physica A 325, 243 (2003).

[4] J.M. Sancho, A. Sanchez, Eur. Phys. J. B 16, 127 (2000); N.R. Quintero, A. Sanchez, F.G. Mertens, Phys. Rev. E 60, 222 (1999).

[5] A.S. Mikhailov, L. Schimansky-Geier, W. Ebeling, Phys. Lett. A 96, 453 (1983); L. Schimansky-Geier, Ch. Zulicke, Z. Phys. B 82, 157 (1991).

[6] F. Marchesoni, Phys. Rev. Lett. 77, 2364 (1996); M.G. Clerc, C. Falconi, E. Tirapegui, Phys. Rev. Lett. 94, 148302 (2005).

[7] F.G. Bass, R. Bakanas, Europhys. Lett. 53, 444 (2001).

[8] R. Bakanas, Phys. Rev. E 69, 016103 (2004).

[9] R. Bakanas, Phys. Rev. E 71, 026201 (2005).

[10] R. Bakanas, Phys. Rev. E 78, 046202 (2008).

[11] A. Raguotis, F. Ivanauskas, R. Bakanas, Phys. Scr. 74, 629 (2006).

[12] R. Bakanas, A. Raguotis, F. Ivanauskas, Phys. Scr. 77, 055003 (2008).

[13] M.C. Cross, P.C. Hohenberg, Rev. Mod. Phys. 65 851 (1993); J. Fort, V. Mendez, Rep. Prog. Phys. 65, 985 (2002); Wim van Saarloos, Phys. Rep. 386, 29 (2003); J. Fort, T. Pujol, Rep. Prog. Phys. 71, 086001 (2008).

[14] G. Abramson, A.R. Bishop, V.M. Kenkre, Phys. Rev. E 64, 066615 (2001); E.P. Zemskov, K. Kassner, S.C. Muller, Eur. Phys. J. B 34, 285 (2003); E.P. Zemskov, K. Kassner, Eur. J. Phys. 25, 361 (2004); E.P. Zemskov, Phys. Rev. E 69, 036208 (2004); V. Mendez, V. Ortega-Cejas, E.P. Zemskov, J. Casas-Vazquez, Physica A 375, 50 (2007).

[15] R. Bakanas, Nonlinearity 16, 313 (2003).

[16] A. Kurganov, P. Rosenau, Nonlinearity 19, 171 (2006).

[17] F. Schlogl, C. Escher, R.S. Berry, Phys. Rev. A 27, 2698 (1983); F.G. Bass, R. Bakanas, Phys. Lett. A 214, 301 (1996); F.G. Bass, R. Bakanas, Waves Random Media 10, 217 (2000).

[18] R. Jackiw, Rev. Mod. Phys. 49, 681 (1977); A.R. Bishop, J.A. Krumhansl, S.E. Trullinger, Physica $D$ 1, 1 (1980).

[19] V. Jasaitis, F. Ivanauskas, R. Bakanas, Nonlinear Anal. Model. Control 13, 433 (2008). 